\title{
Assessment of the Effect of Transcranial Direct Current Stimulations (tDCS) in Focused Attention Enhancement Using Event-Related Potentials
}

\author{
Shokoufeh Moezzi ${ }^{1}$, Majid Ghoshuni ${ }^{*}$, Mahdi Amiri ${ }^{2}$ \\ ${ }^{1}$ Department of Biomedical Engineering, Mashhad Branch, Islamic Azad University, Mashhad, Iran \\ ${ }^{2}$ Department of psychology, Mashhad University of Medical Science, Mashhad, Iran
}

Article Info:

Received: 20 June 2020

Revised: 23 July 2020

Accepted: 27 Sep 2020

\section{A BSTRACT}

Introduction: Attention is a multi-dimensional cognitive process. Transcranial direct current stimulation (tDCS) is an effective approach for the improvement of attention. Using Eventrelated potentials (ERP) frequency components, the main goal of this study was to develop an attention index to assess improvement of attention in healthy people by applying tDCS. Materials and Methods: 13 subjects (6 males and 7 females, with mean age $24.53 \pm 4.5$ years) participated in this study. First, participants were asked to fill out the Adult ADHD self-report scale, Beck's depression, and Spielberger anxiety questionnaires. Simultaneous to recording their brain signals (19 channels), subjects were asked to participate in the Integrated visual and auditory (IVA) test. This test entails 500 stimulations, which include 125 auditory targets, 125 visual targets, 125 auditory non-target, and 125 visual non-target stimulations lasting for 13 minutes, after recording the electroencephalogram (EEG), the Dorsolateral Prefrontal Cortex (DLPFC) in subjects' left hemisphere is stimulated using tDCS, with $1 \mathrm{~mA}$ current for 20 minutes. The stimulation period was 5 consecutive days. To analyze the data, after removing noise and artifacts, ERP components were extracted using synchronous averaging. Then frequency domains feature in every stimulation type were extracted from ERP components. Correlation between percentage changes and the subject's IVA test results were calculated before and after applying tDCS. Results: The result showed that Beta energy significantly increased after applying tDCS. Furthermore, a significant correlation was observed between beta energy and auditory omission error in FP1 channel. Conclusion: tDCS decreases omission error and increases beta energy in auditory stimulation, which then leads to the improvement of focus attention. Further studies are needed to assess the effect of tDCS on the improvement of attention in auditory attention deficit.

\section{Keywords: \\ 1. Attention \\ 2. Transcranial Direct \\ Current Stimulation}

3. Surveys and Questionnaires

*Corresponding Author: Majid Ghoshuni

E-mail: ghoshuni@mshdiau.ac.ir 


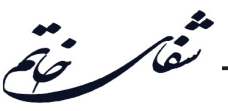

\title{
ارزيابى تحريك جريان مستقيم فراجمجمهاى در افزايش توجه متمركز با استفاده از يتانسيلهاى رخداد وابسته به رخداد
}

\author{
شكوفه معزّى'، مجيد قشونى"، مهدى اميرى'

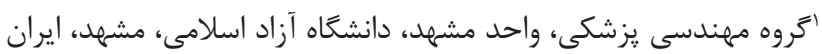

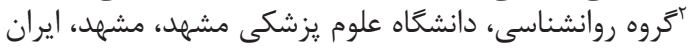

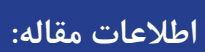

بذيرش: و مهر 9941

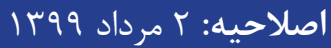

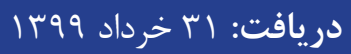

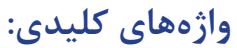

(1)

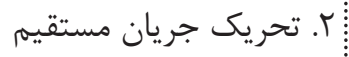

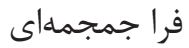

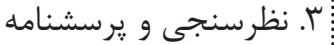

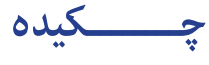

مقدمه: توجه يك فرآيند شناختى تجند بعدى است. تحريك جريان مستقيم فرا جمجمالى (tDCS)

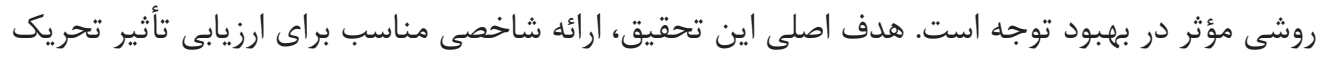

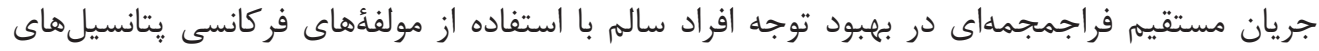

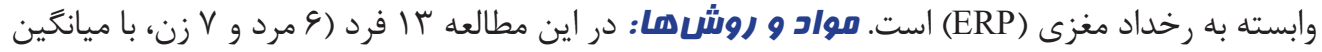

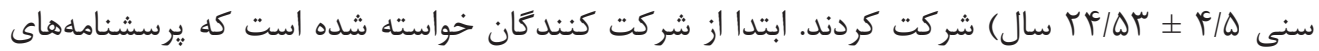

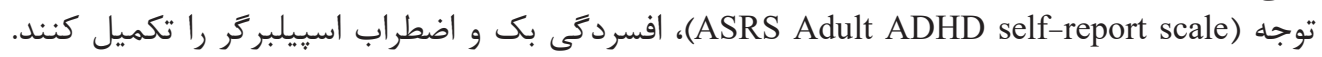

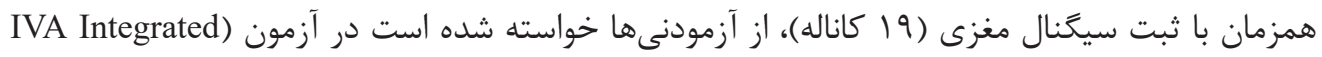
(Visual and Auditory

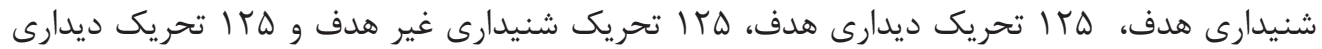

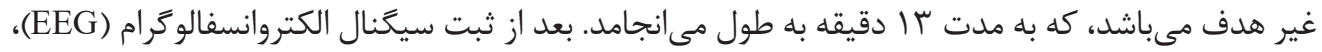

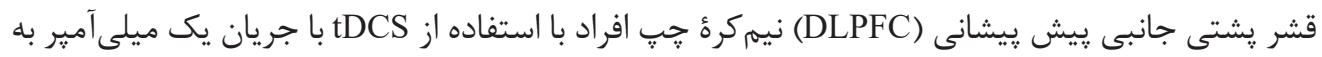

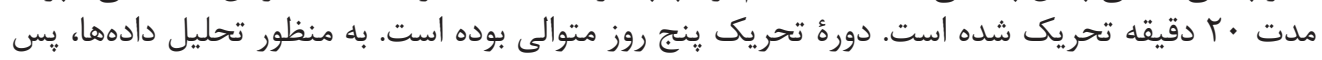

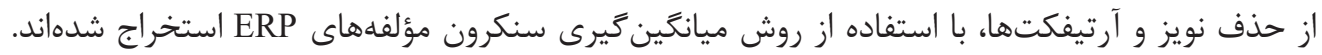

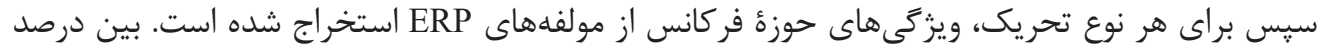

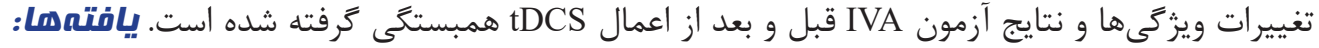

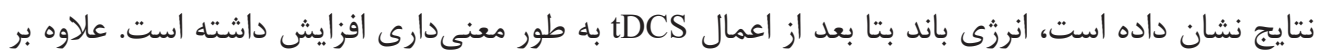

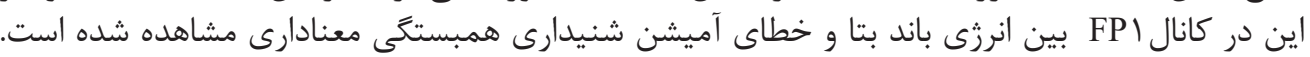

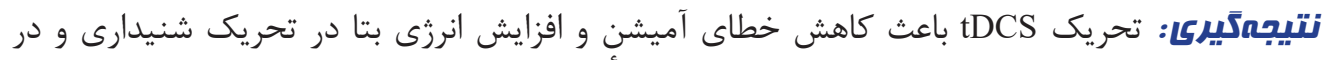

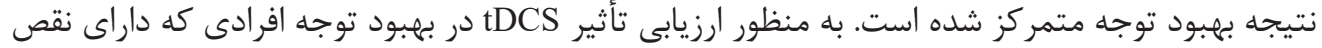

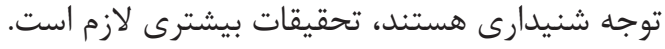

$$
\text { *ويسنده مسئول: مجيد قشونى }
$$




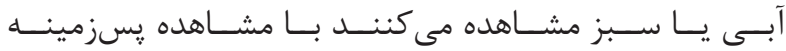

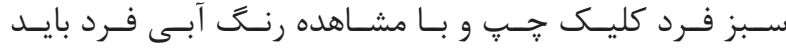

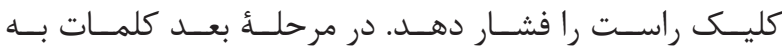

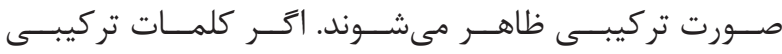

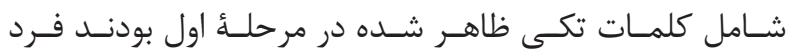

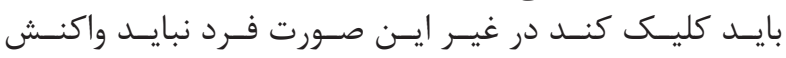

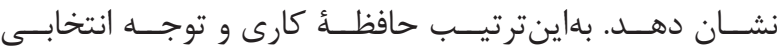

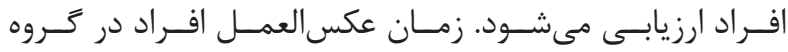

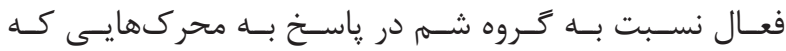

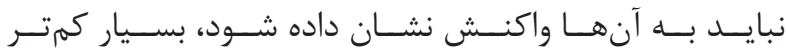

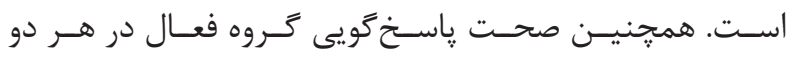

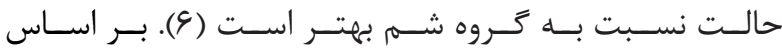

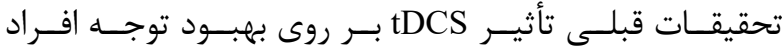

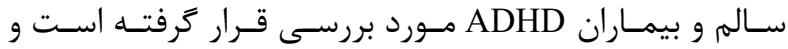

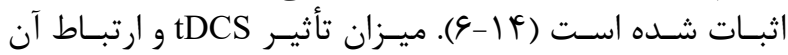

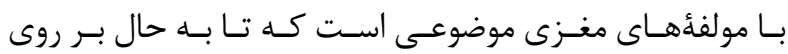

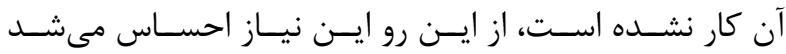

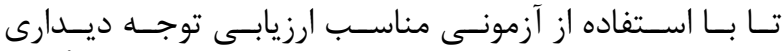

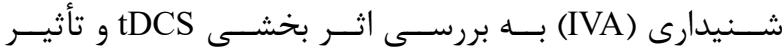

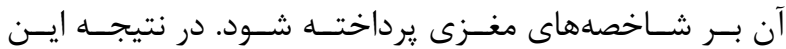

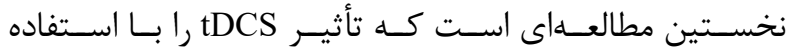

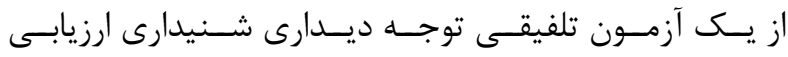

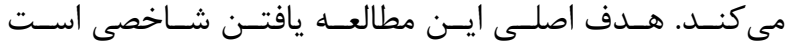

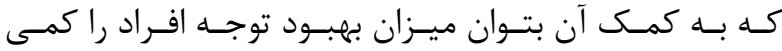

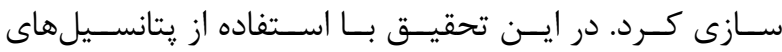

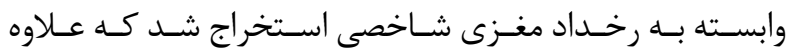

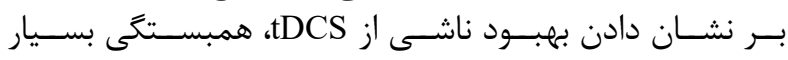

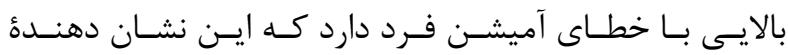

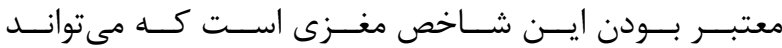

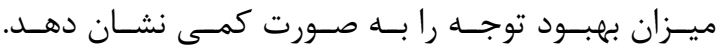

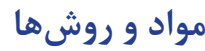

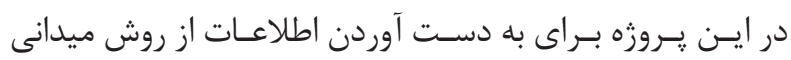

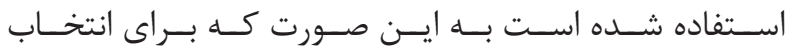

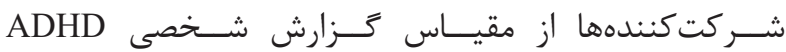

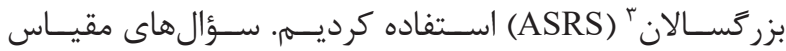
شSRS

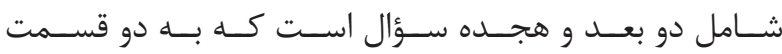

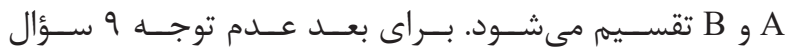

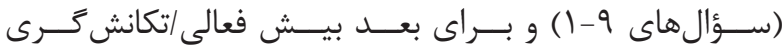

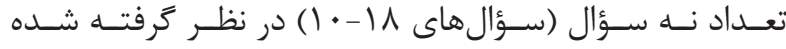

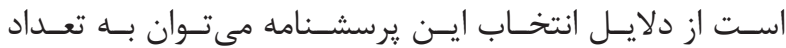

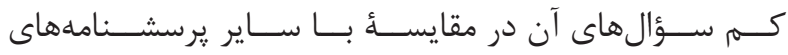

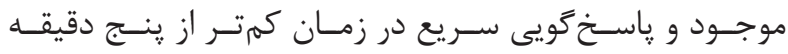

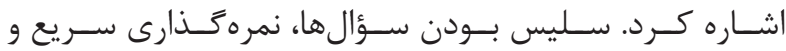

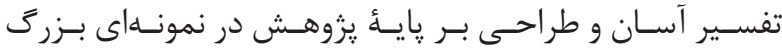

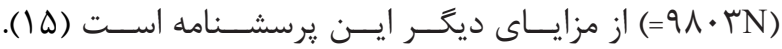

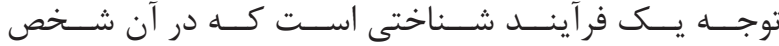

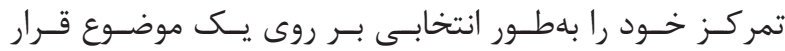

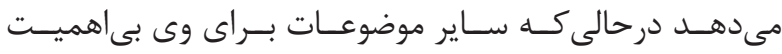

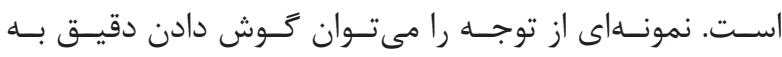

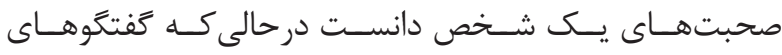

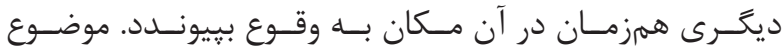

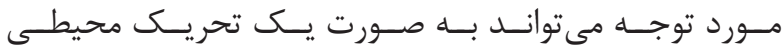

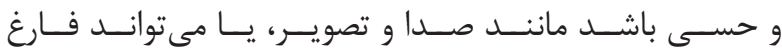

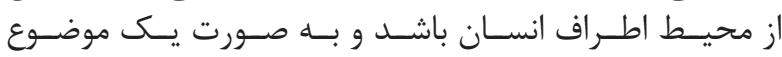

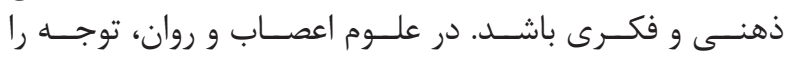

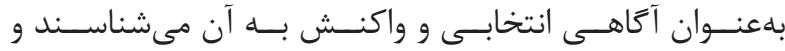

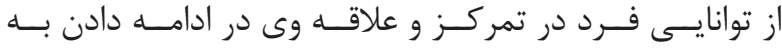

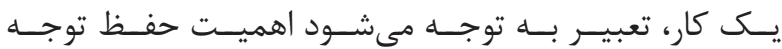

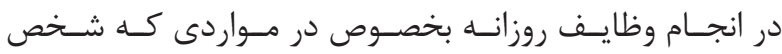

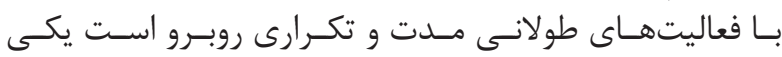

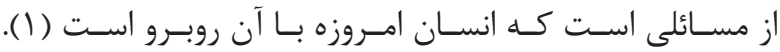

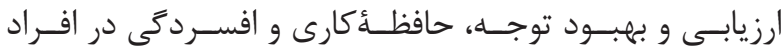

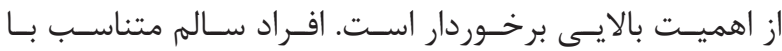

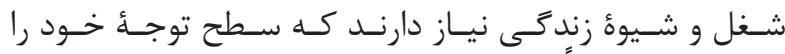

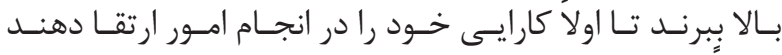

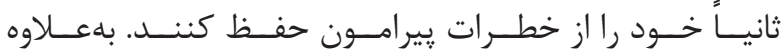

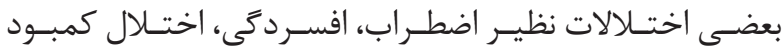

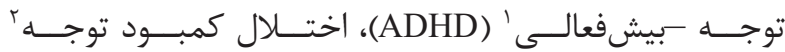

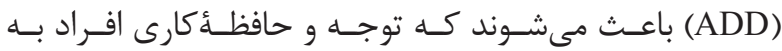

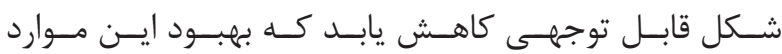

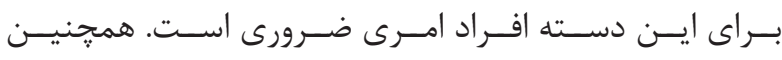

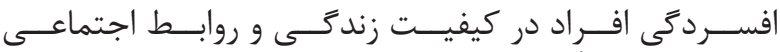

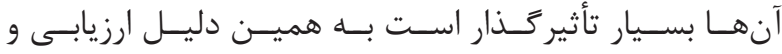

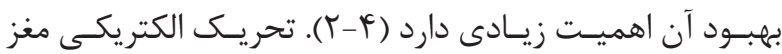

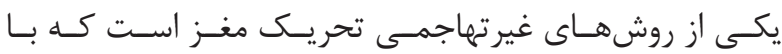

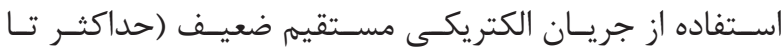

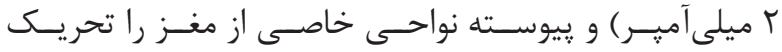

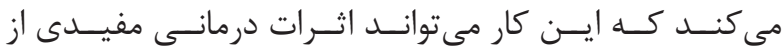

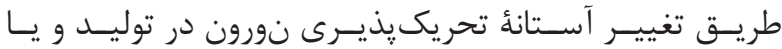

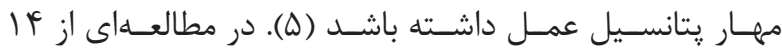

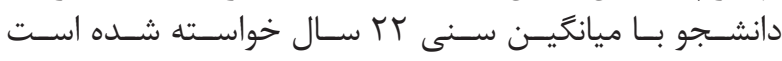

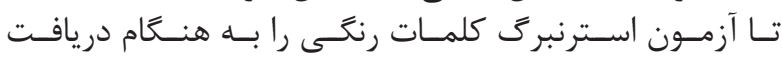

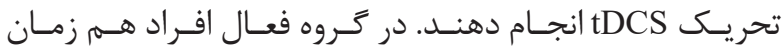

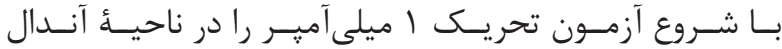

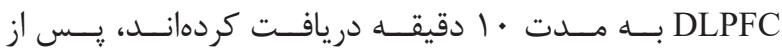

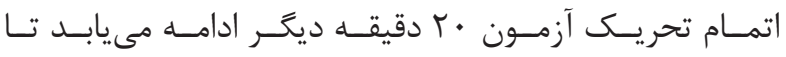

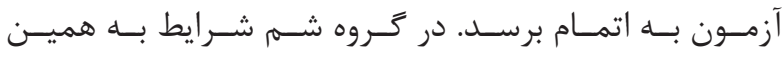

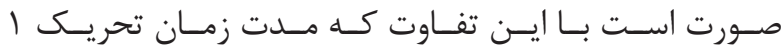

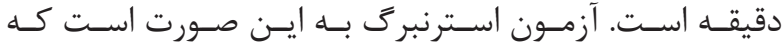

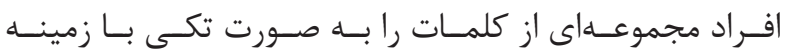

${ }^{3}$ Adult Self-Report Scale; ASRS 


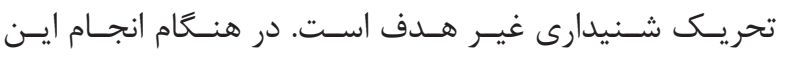

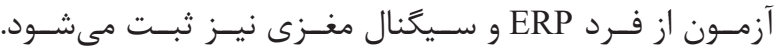

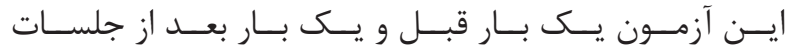

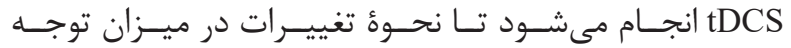

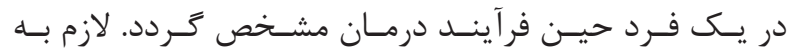

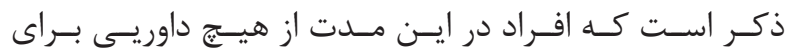

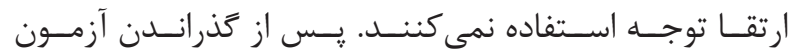

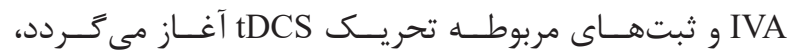

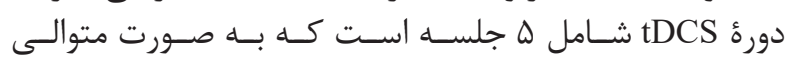
بركـزار مى شـود تحريـك آنـد در ناحيـهـ جــ

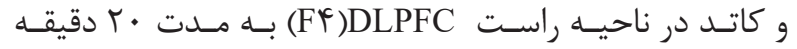

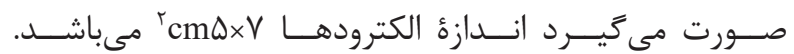

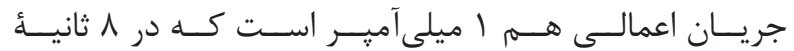

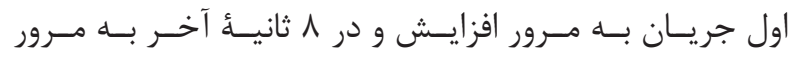

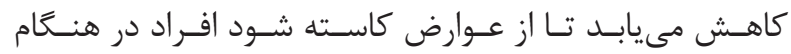

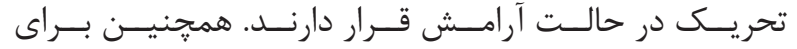

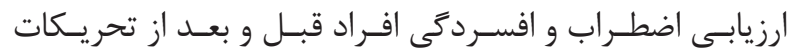

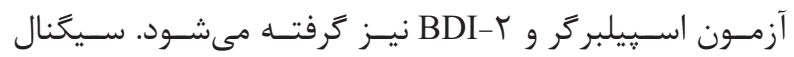

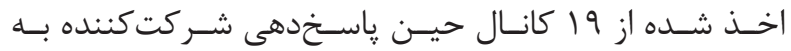

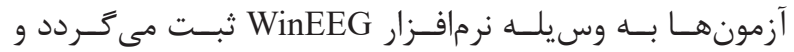

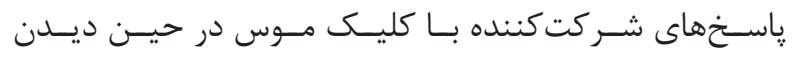

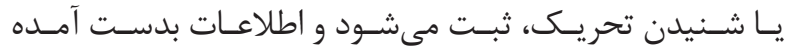

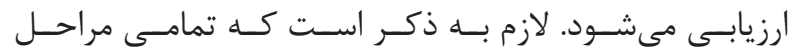

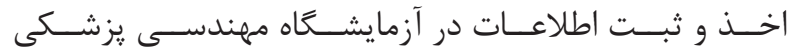

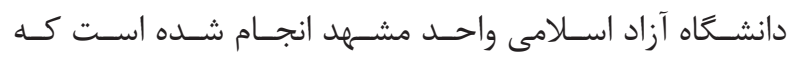

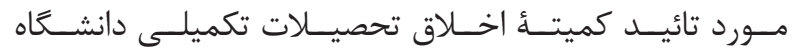

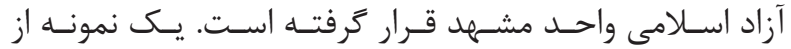

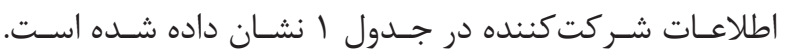

همجنيـن در مقالـهاى كارايسى نسـخه فارسـى آن در تشـخـيص

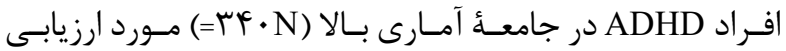

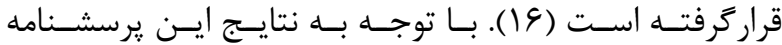

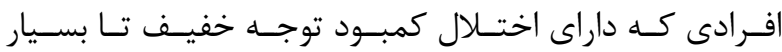

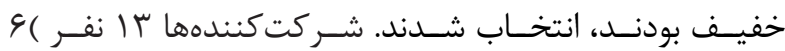

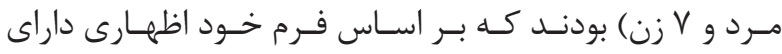

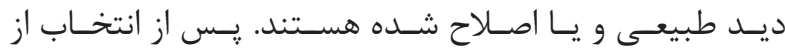

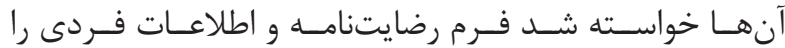

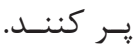
بافته ها

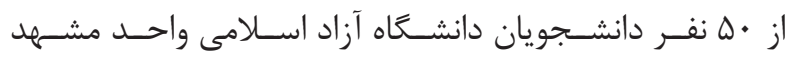

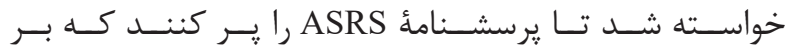

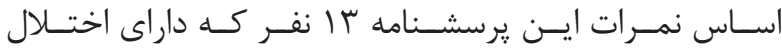

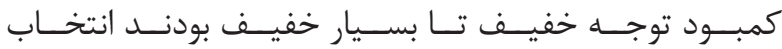

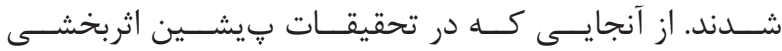

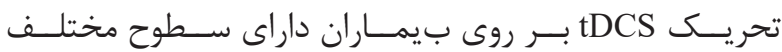

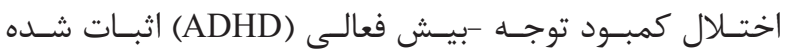

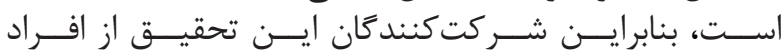

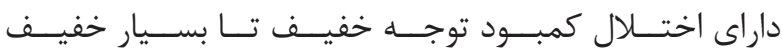

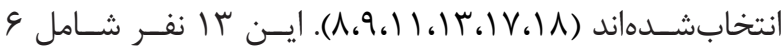

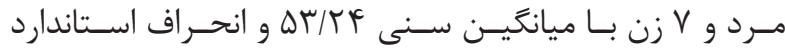

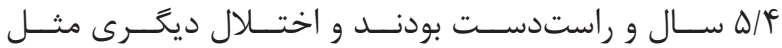

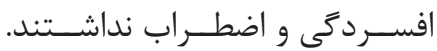

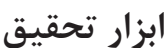

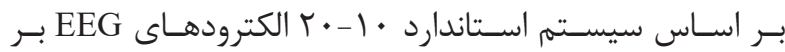

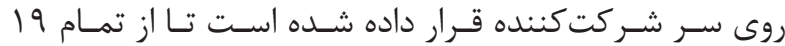

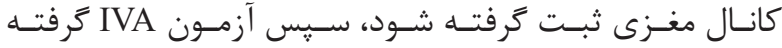

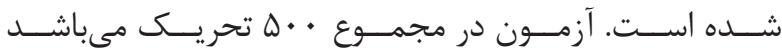

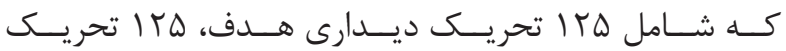

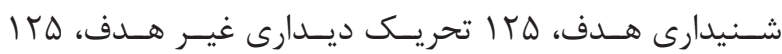

جدول ا- كزارش اوليه از پاسخدهى شركت كنندكان به آزمون

\begin{tabular}{|c|c|c|c|c|c|}
\hline واريانس زمان عكس العمل & زمان عكسالعمل & خطا كاميشن & خطا آميشن & 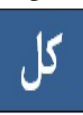 & نام كروه \\
\hline$\cdot / \cdot \mu$ & $\cdot A V Y$ & . & If & lis & تحريك هلف ديلارى \\
\hline$\cdot / \psi^{k}$ & $\cdot / 991$ & . & $r$ & lita & تحريك هلدف شنيدارى \\
\hline . & . & V & . & lis & تحريك غير هلف شنيدارى \\
\hline . & . & 19 & & lis & تحريك غير هلف ديلارى \\
\hline
\end{tabular}




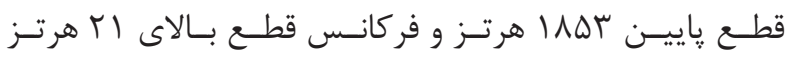

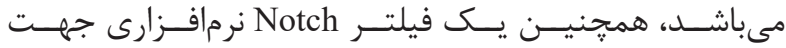

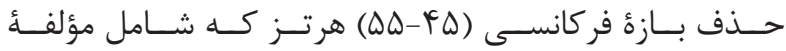

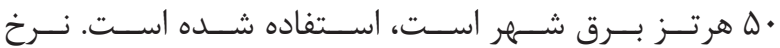

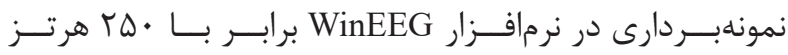

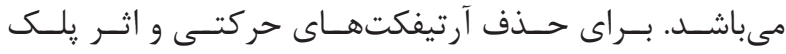

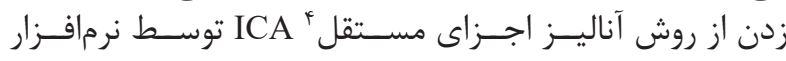
WinEEG

\section{استخراج ويثزَى ها}

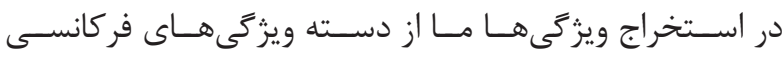

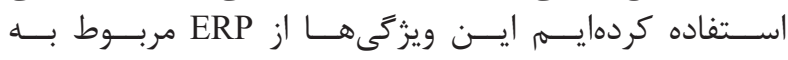

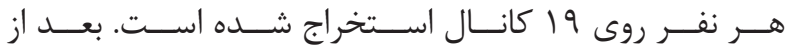

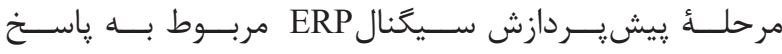

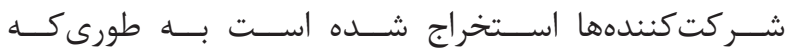

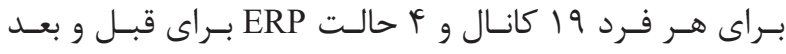

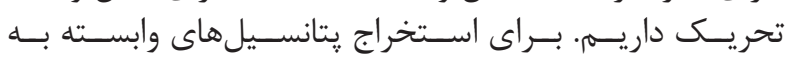

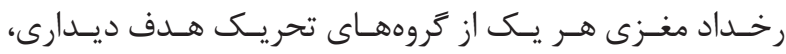

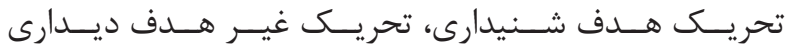

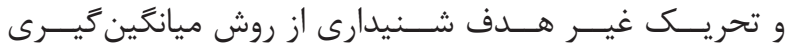

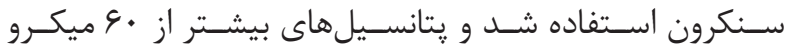

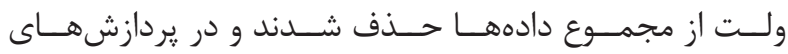

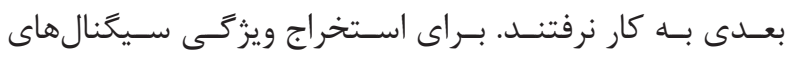

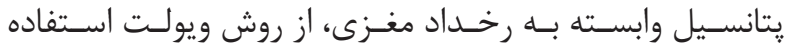

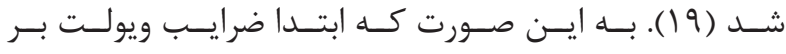

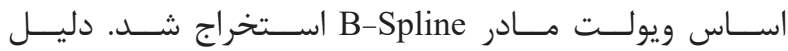

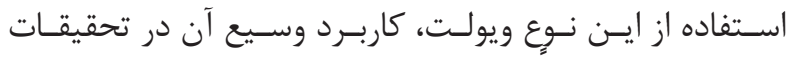

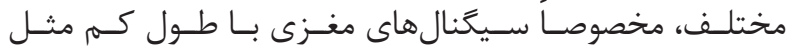

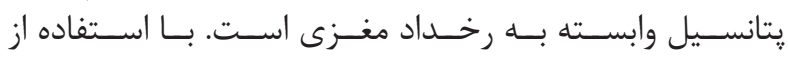

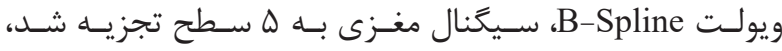

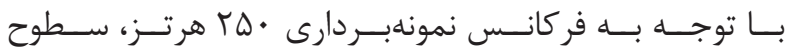

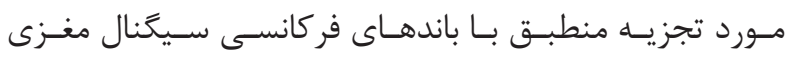

\section{جلسات تحريك}

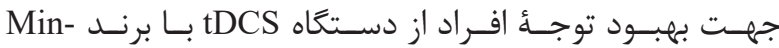

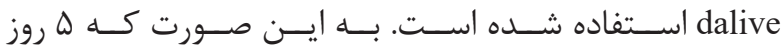

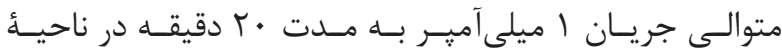

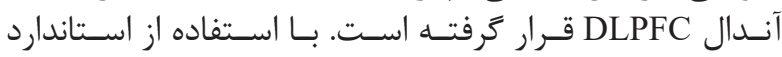

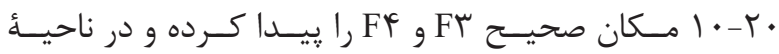

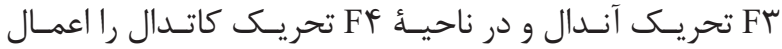
كرديـه.

\section{نحوه اخذ دادهها}

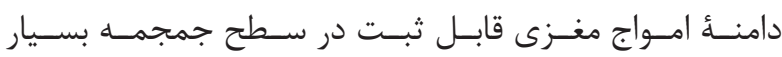

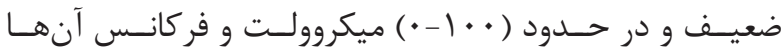

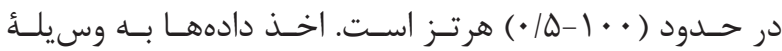

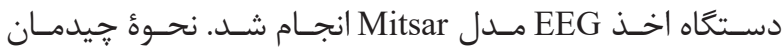

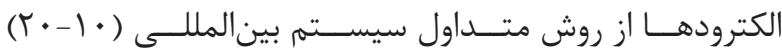

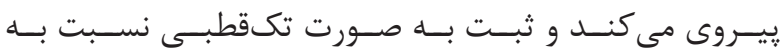

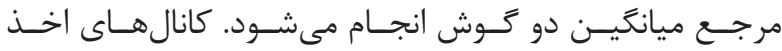
Fpl -Fpr -Fr -FF -Cr -CF -Pr -PF -Ol

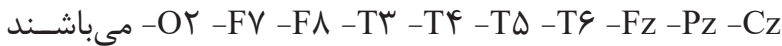

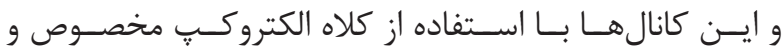

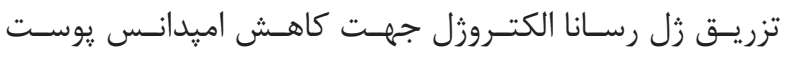

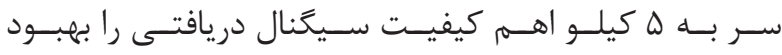

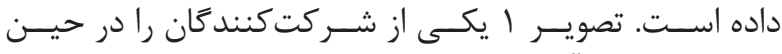

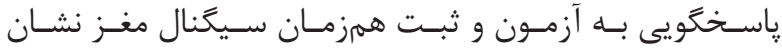

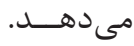

روش تحليل دادهها ييش ير دازش سيخنال

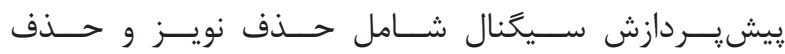

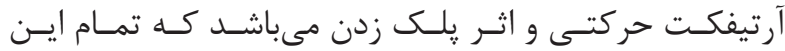

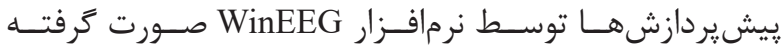

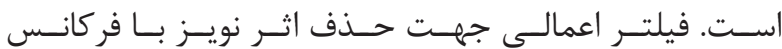

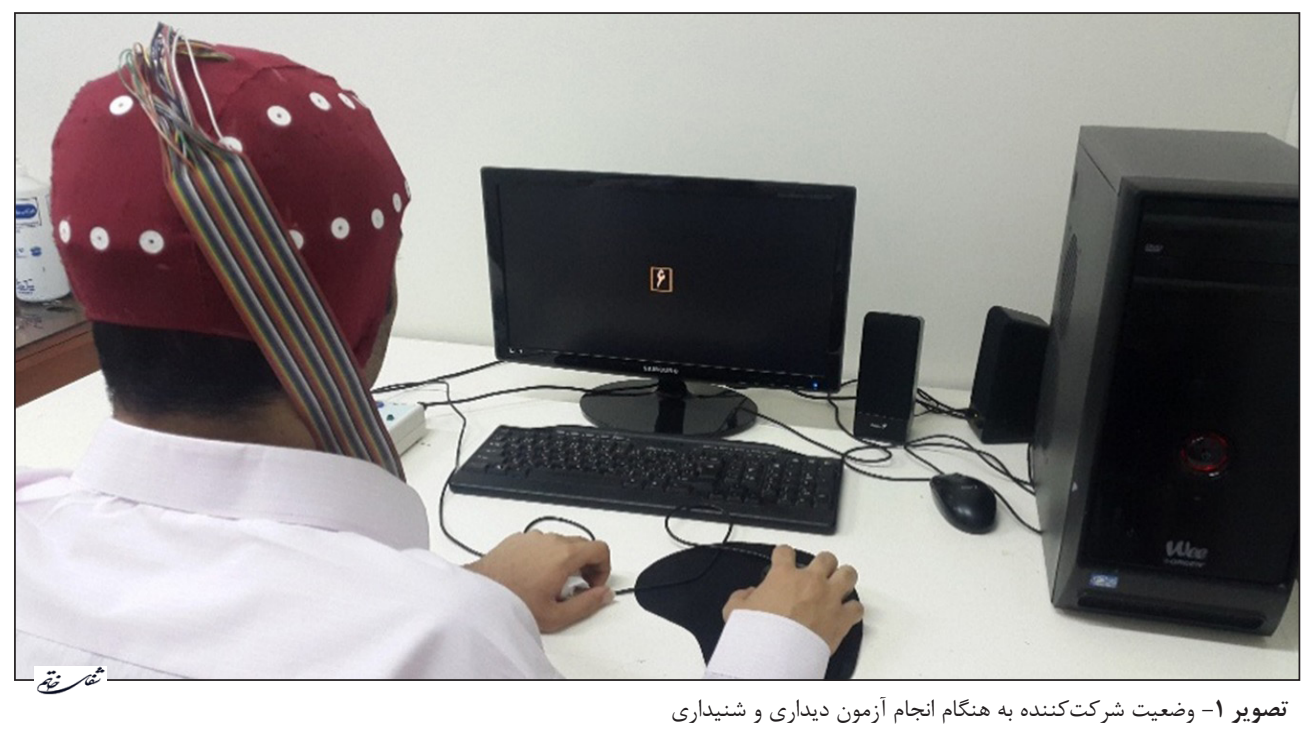

${ }^{4}$ Independent Component Analysis; ICA 


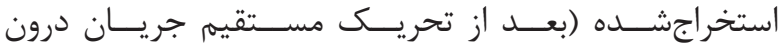

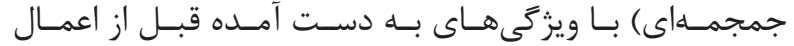

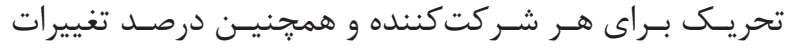

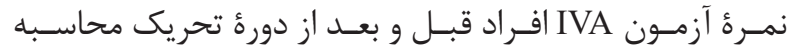

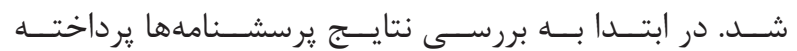

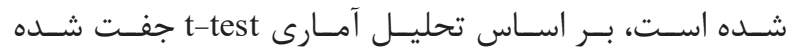

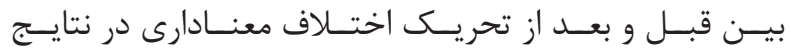

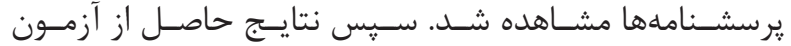

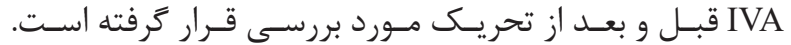

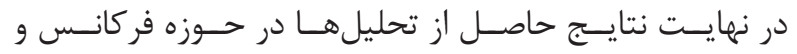

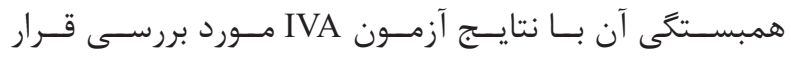

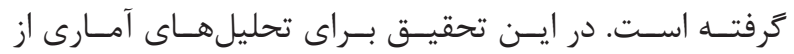

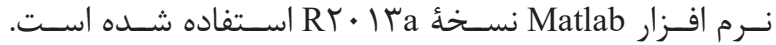

\section{نتايج يرسشنامة}

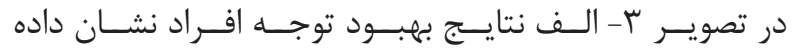

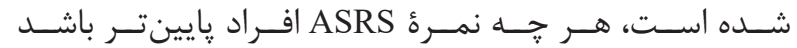

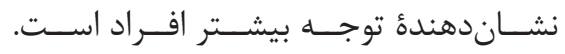

نتايج يرسشنامه r BDI-

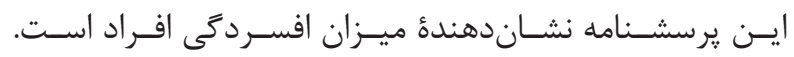

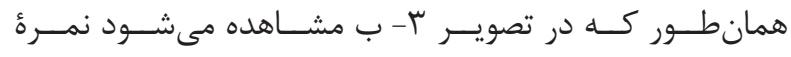

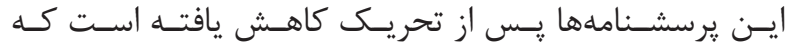

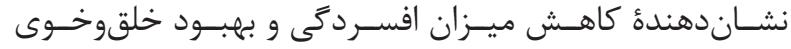

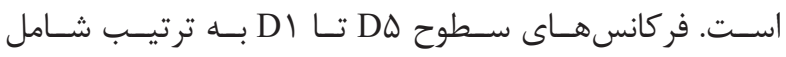

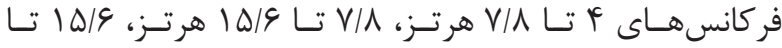
سا

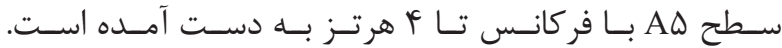

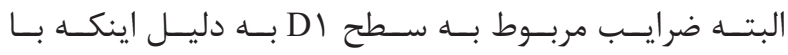

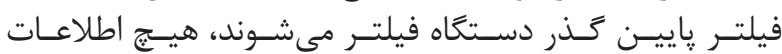

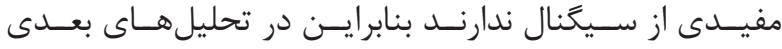

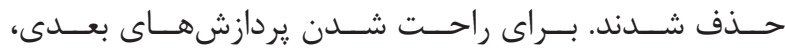

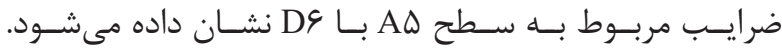

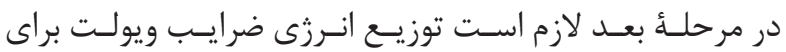

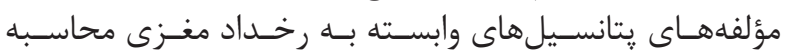

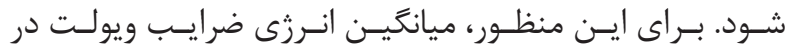

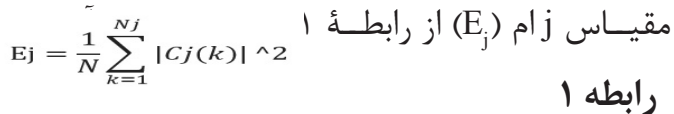

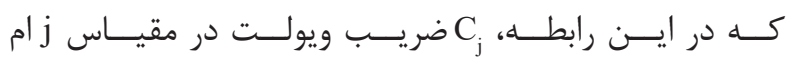

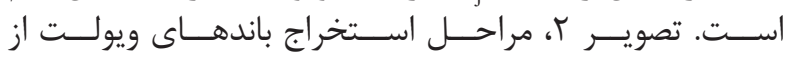

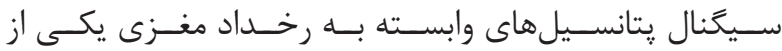

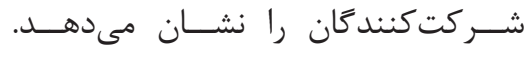

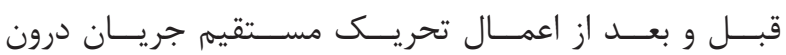

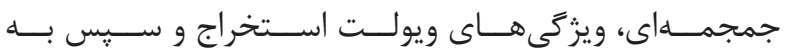

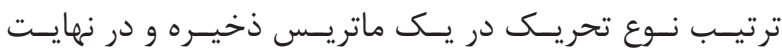

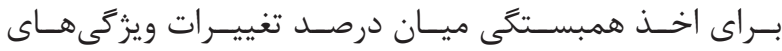

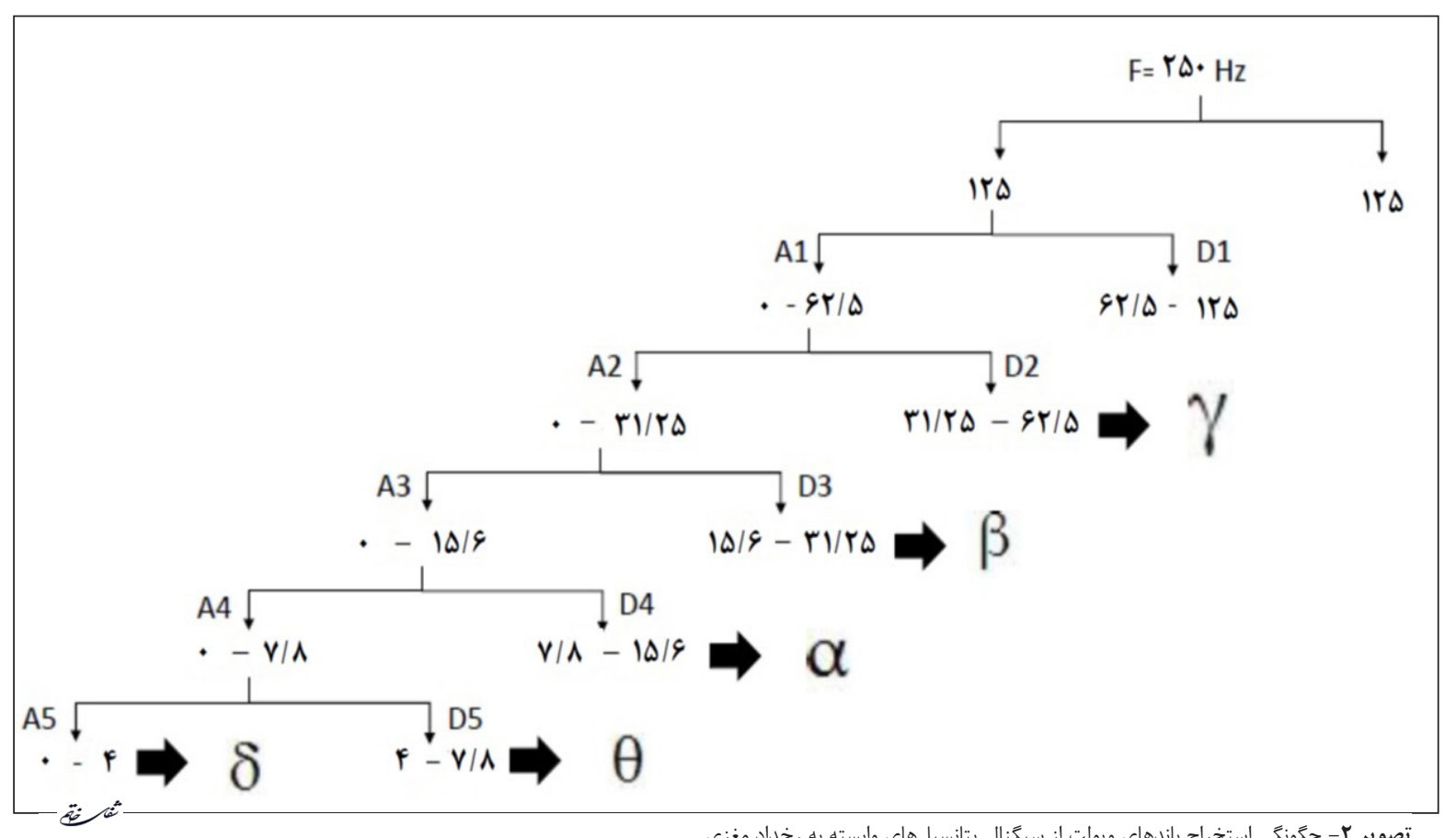




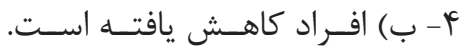

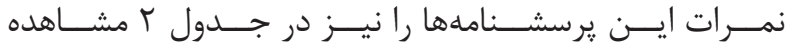

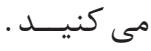

IVA نتايج آزمون

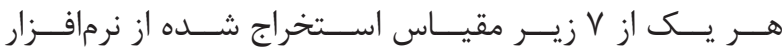

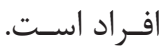

نتايج يرسشنامة اسييلبر

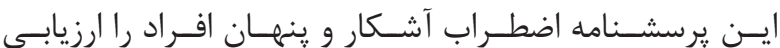

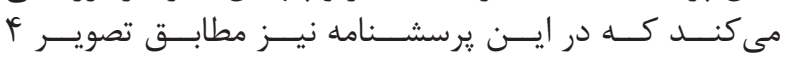

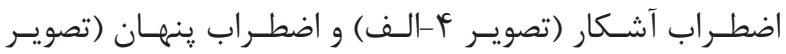

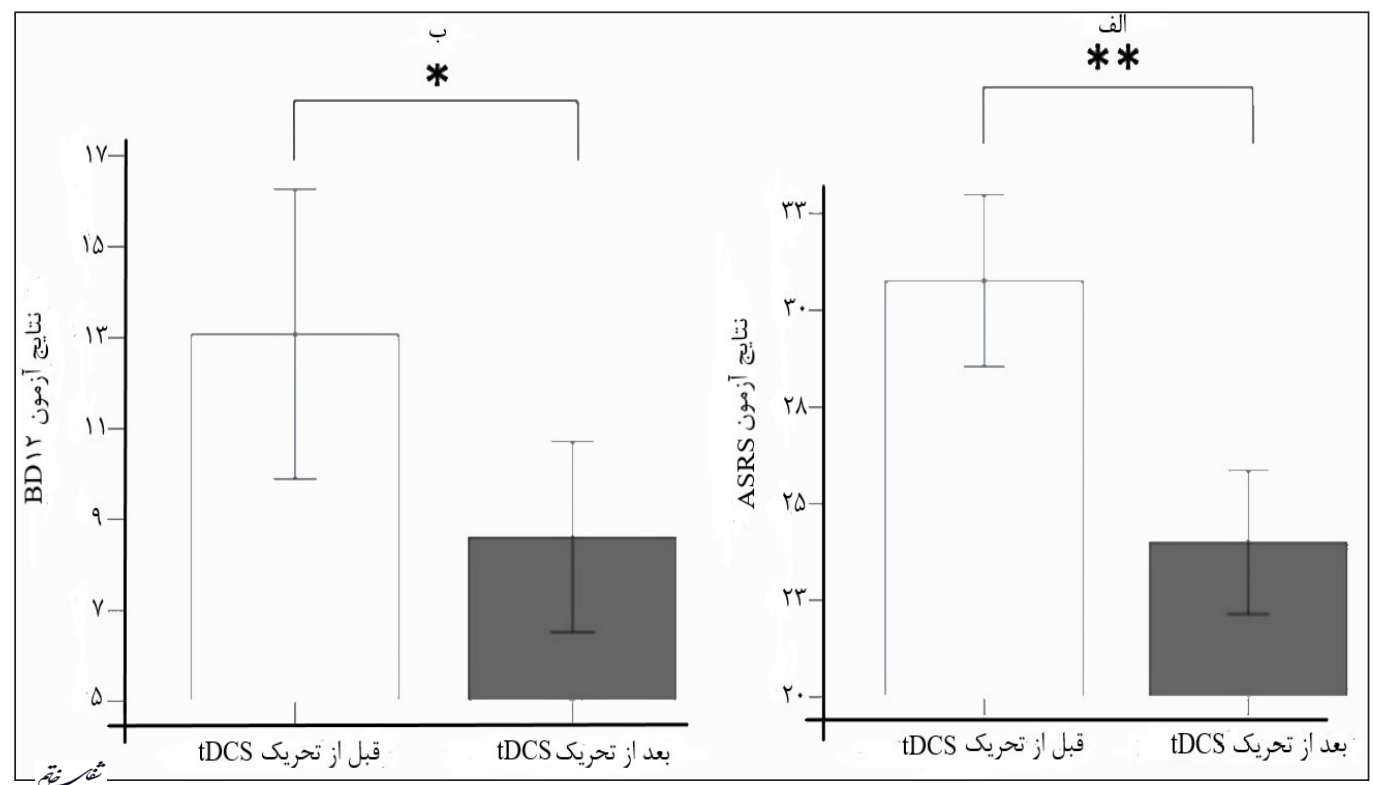

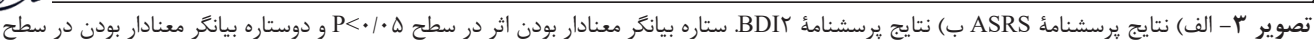

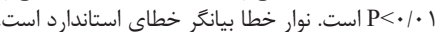

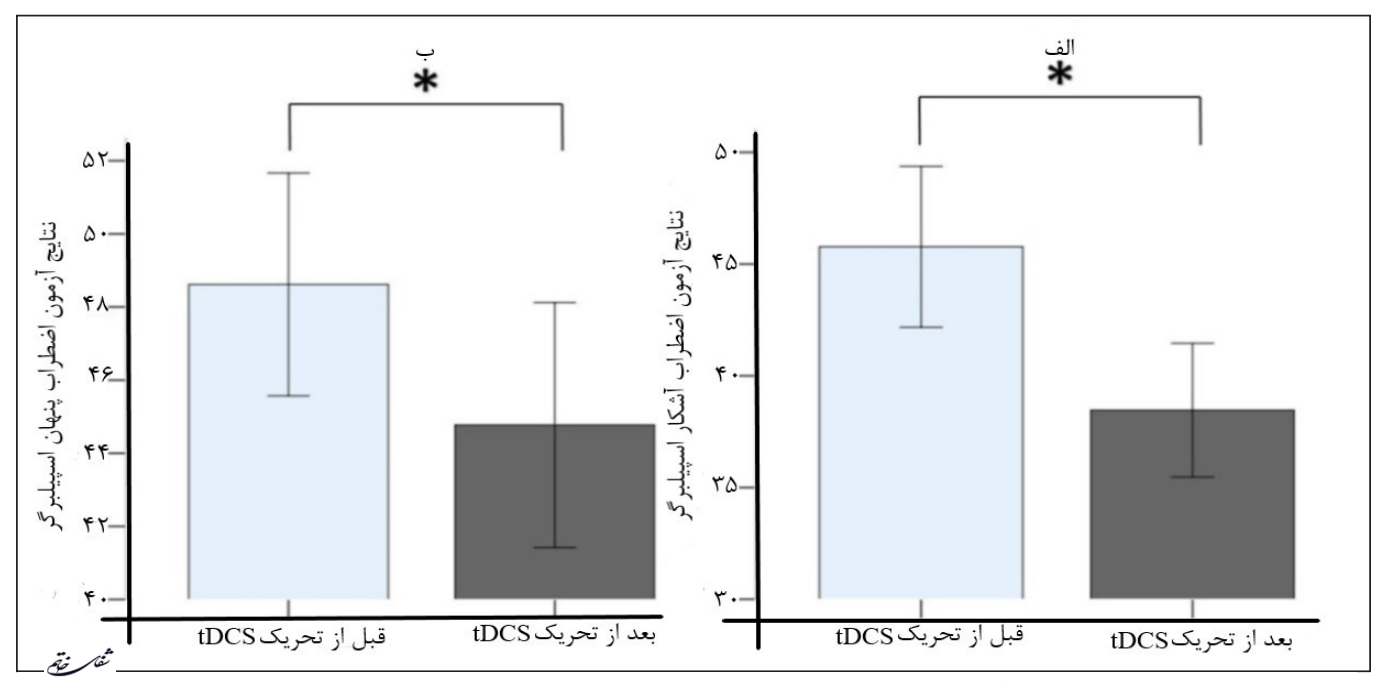

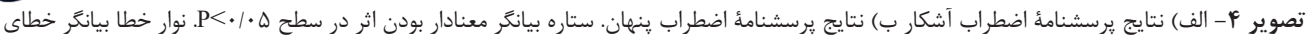


جدول r- نتايج يرسشنامهها

\begin{tabular}{|c|c|c|c|c|c|c|}
\hline t-value & P-value & انحراف استاندارن & ميانكين نمره افراد & انحراف استاندارد از تحريك & قيانكين نمره افراد & يرسشنامه \\
\hline $\mathrm{r} / q \Lambda \mathrm{f}$ & $\cdot 1 \cdot r$ & $9 / 99$ & $r r / .$. & $N \cdot r$ & $r \cdot / V V$ & توجه ASRS \\
\hline r/VqG & $.1 \cdot 19$ & $V / \Delta \%$ & $\mathrm{~N} / \mathrm{GT}$ & $11 / 49$ & $\| \pi / \cdot 1$ & افسردكى بك r \\
\hline r/VYq & $\cdot 1 \cdot 11$ & $1 \cdot 11$. & TN/FG & $1 \% / 91$ & $F \Delta / V V$ & اضطراب آشكار \\
\hline T/TIT & $.1 \cdot F V$ & $\mid r / \cdot V$ & $F F / V$ & $1 \cdot / 91$ & FNGT & اضطراب ينهان اسبيلبركر \\
\hline
\end{tabular}

بحث و نتيجه

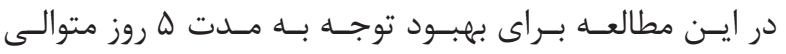

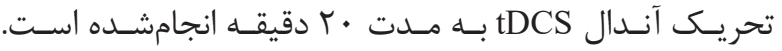

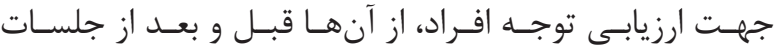

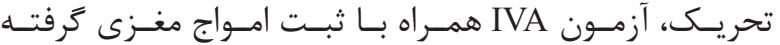

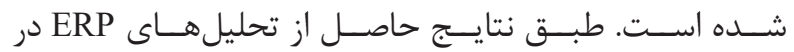

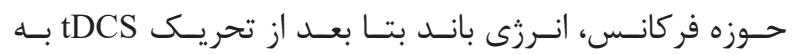

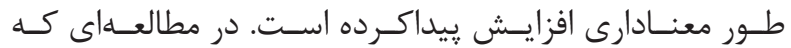

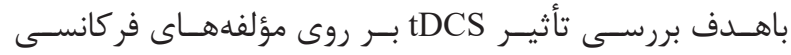

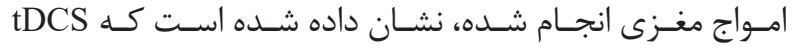

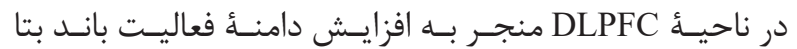

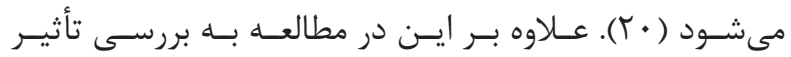

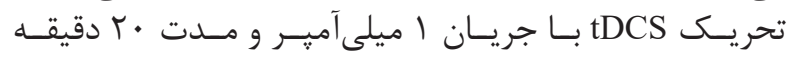

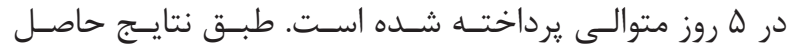

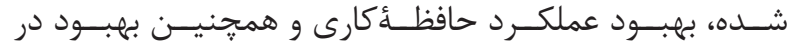

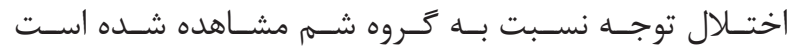

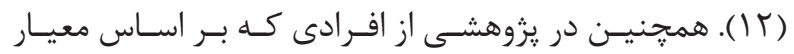
شت اختـلال ADSM-VI

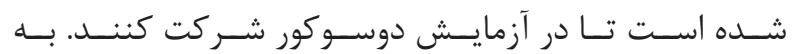

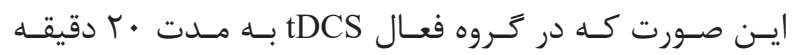
جريـان ا ميلى آميـر تحريـك آنـدى در ناحيـهـ جــ
WinEEG

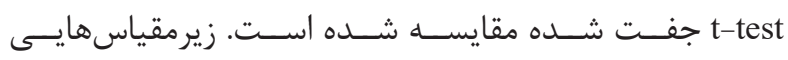

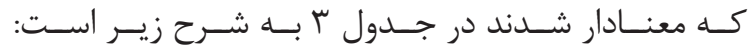

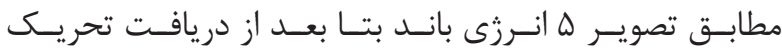
دDCS

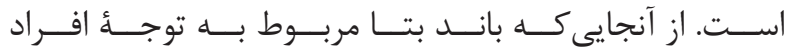

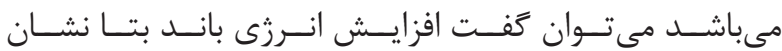

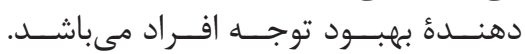

نتايج حاصل از تحليل ويولت در تحريك شنيدارى هدف

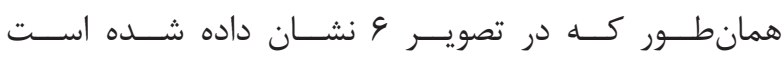

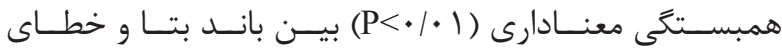

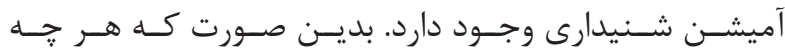

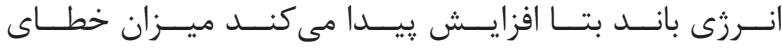

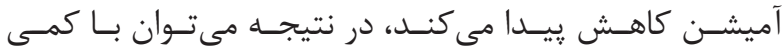

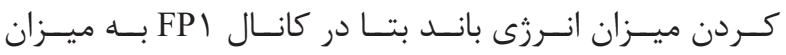

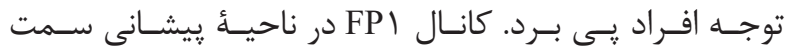

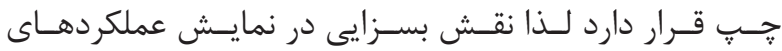

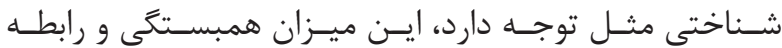

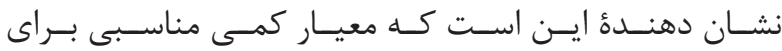

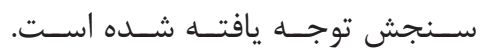

حدول ب- زي مقياس هاي معنادار آزمون IVA

\begin{tabular}{|c|c|c|c|c|c|}
\hline انحراف استاندارد & بعدازئن تحريك & قبر اف أنتاندارد & قبل از تحريى & P-Value & زير مقياس آزمون IVA \\
\hline$\Delta / 4\rangle$ & $\Delta / 9 T$ & $1 \cdot 10 \Delta$ & $1 \pi / 10$ & $\cdot \cdot \cdot \Delta r$ & خطاى آميشن شنيدارى \\
\hline$\Delta / 9$. & $\Delta / 99$ & $11 / \cdot r$ & $11 / 94$ & $\cdot / \cdot \Delta \Delta$ & خطاى كاميشن شنيدارى \\
\hline$Y / T \Lambda$ & $\psi / \cdot \cdot$ & $1 \pi / 9$. & $1 \cdot 1 / \Delta$ & $\cdot \cdot \cdot \mid \Delta r$ & خطاى كاميشن ديدارى \\
\hline 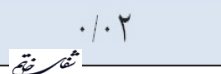 & $\cdot / \cdot r$ & $\cdot / \cdot r$ & $\cdot 1 \cdot 4$ & $\cdot / \cdot r \cdot q$ & واريانس زمان عكسالعمل شنيدارى \\
\hline
\end{tabular}

${ }^{5}$ Diagnostic and Statistical Manual of Mental Disor 


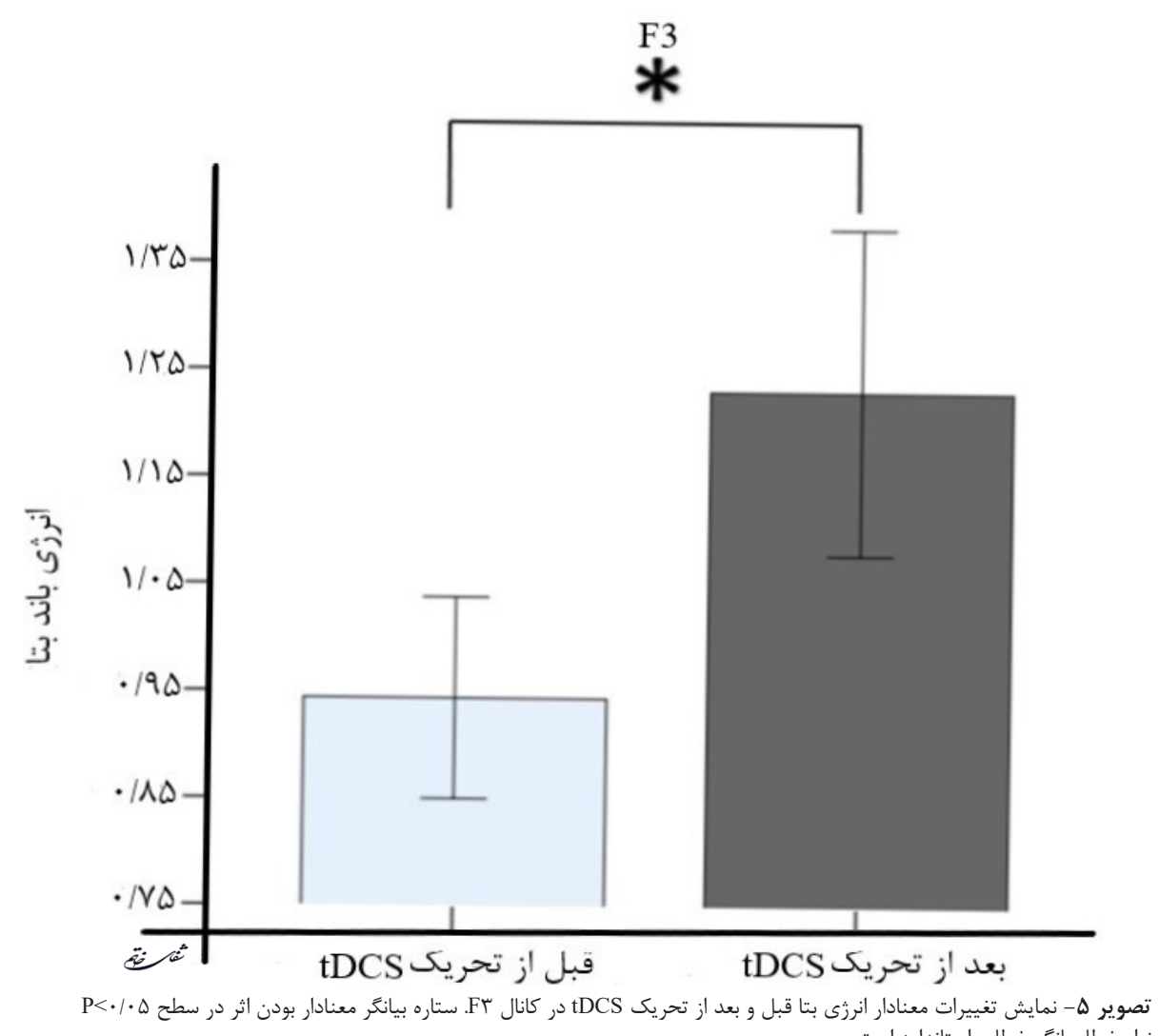

FP1

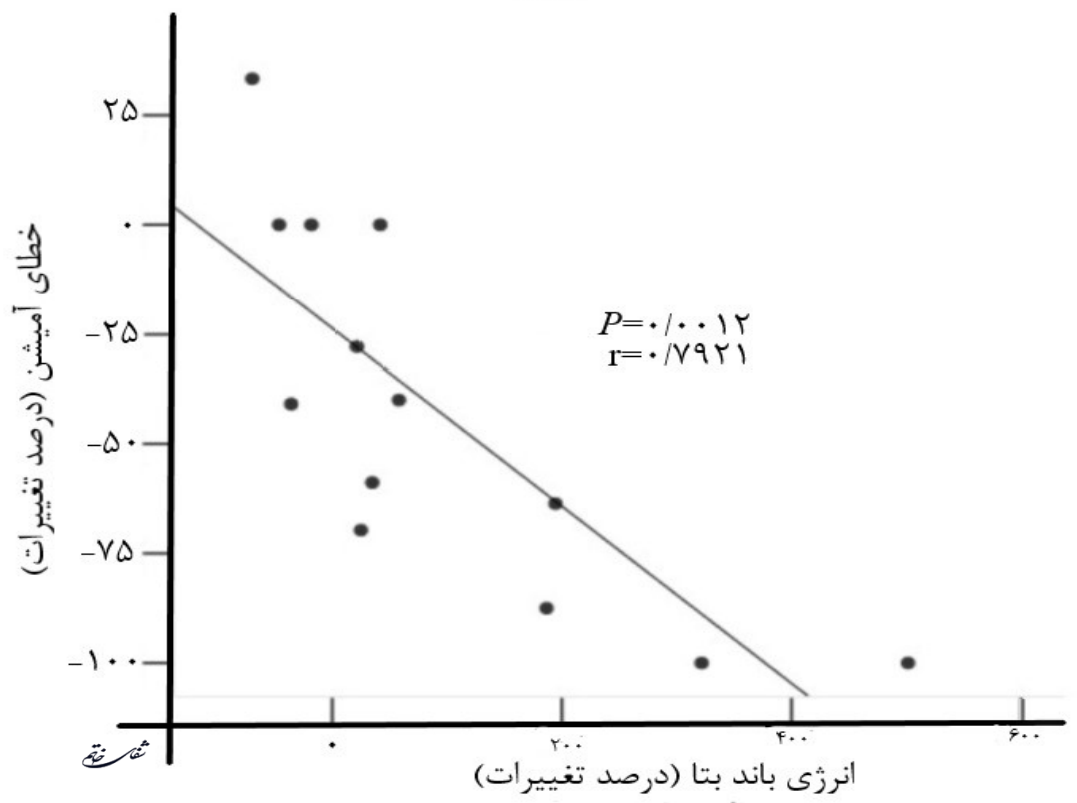

FPI تصوير 9- همبستخى بين درصد تغييرات انرزى بتا و درصد تغييرات خطاى آميشن در تحريك شنيدارى هدف در كانال

افـراد سـالم كار شــده اسـت. شـركت كنند

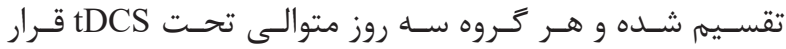

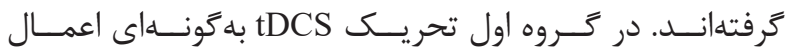

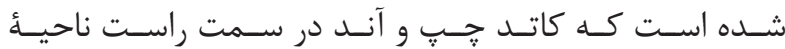

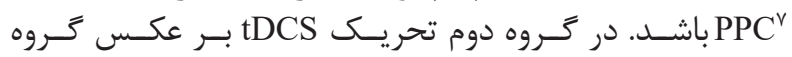

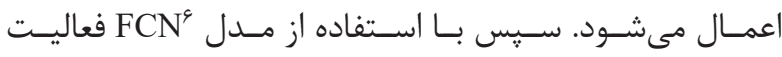

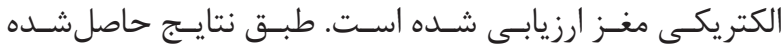

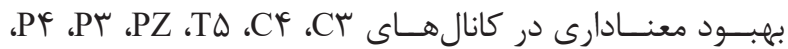

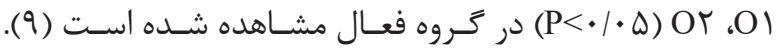

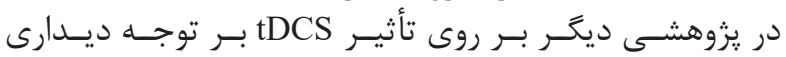

${ }^{6}$ Functional cortical network

${ }^{7}$ Posterior parietal cortex 
ASRS

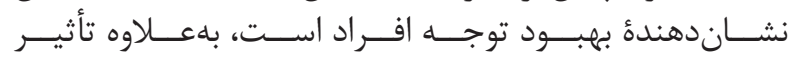

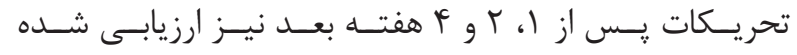

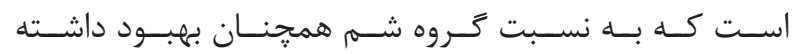

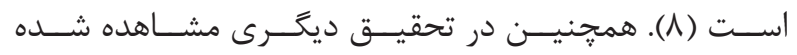

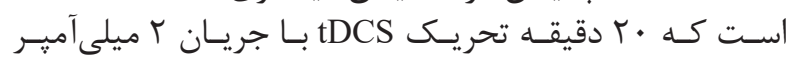

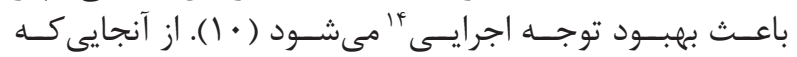

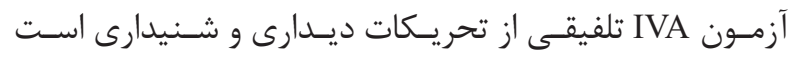

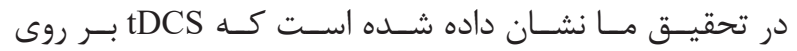

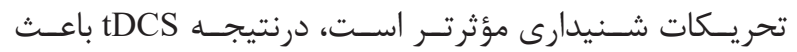

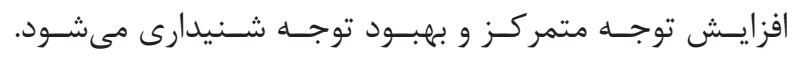

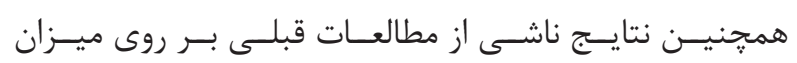

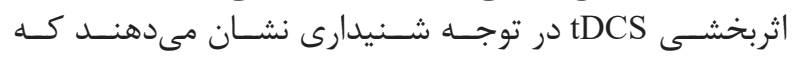

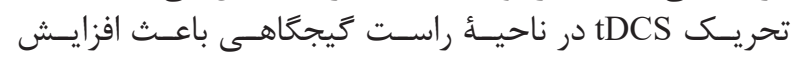

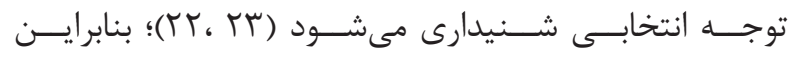

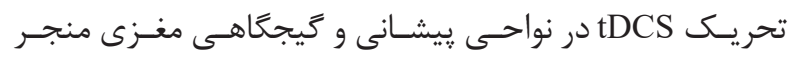

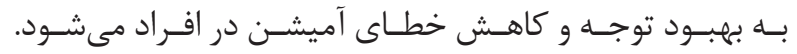

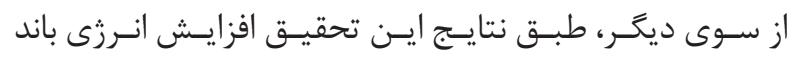

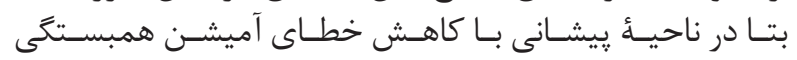

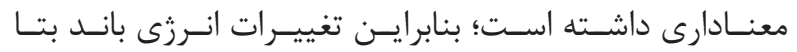

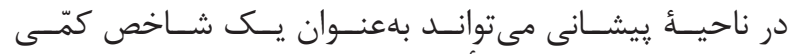

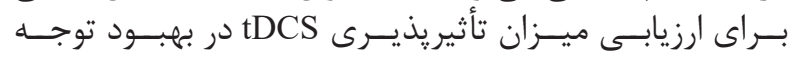

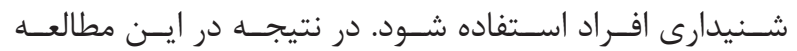

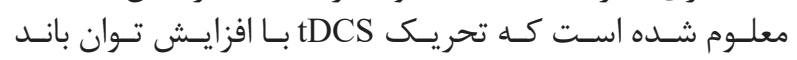

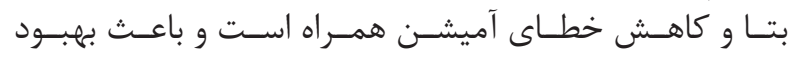

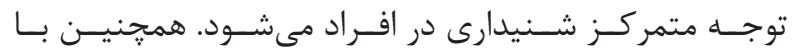

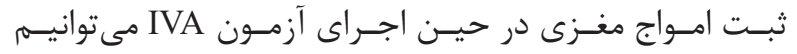

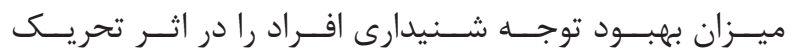
tDCS كمّسى سـازى كنيـهم.

1. Azarnoosh M. Variability of Non-Linear Dynamic Parameters of EEG Signal in Attentive Long-Term Activity: Islamic azad university; 2011.

2. Bush GJN. Attention-deficit/hyperactivity disorder and attention networks. Neuropsychopharmacology. 2010; 35(1): 278-300.

3. McClure EB, Monk CS, Nelson EE, Parrish JM, Adler A, Blair RJR, et al. Abnormal attention modulation of fear circuit function in pediatric generalized anxiety disorder. Arch Gen Psychiatry. 2007; 64(1): 97-106.

4. Amir N, Beard C, Burns M, Bomyea JJJoap. Attention modification program in individuals with generalized anxiety disorder. Abnormal Psychology. 2009; 118(1): 28-33.

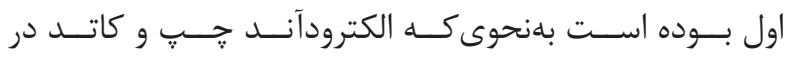

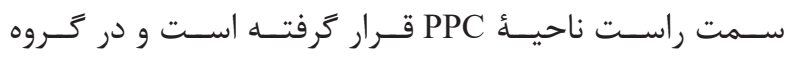

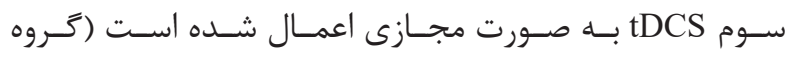

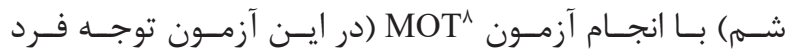

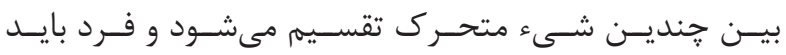

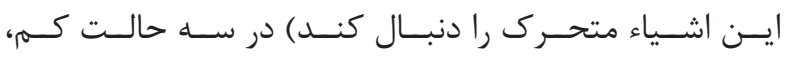

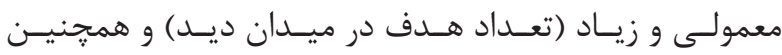

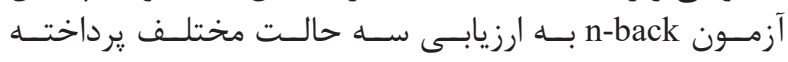

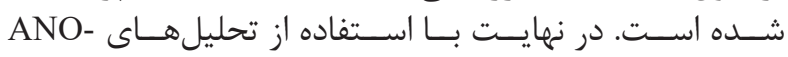

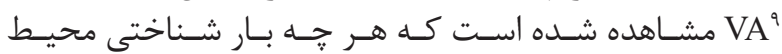

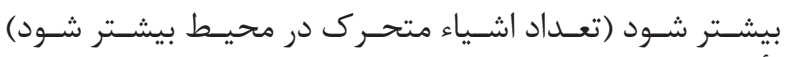

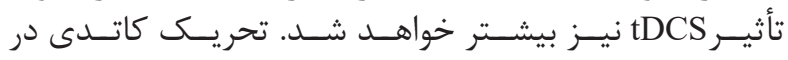

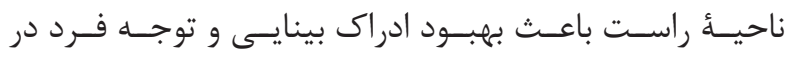

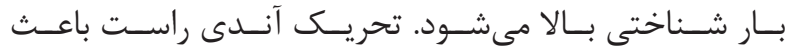

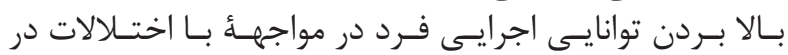

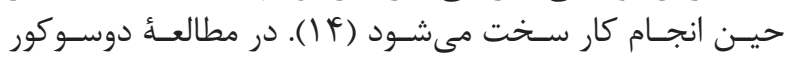

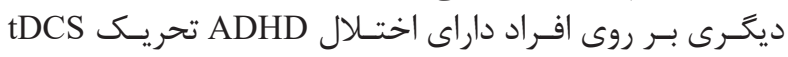

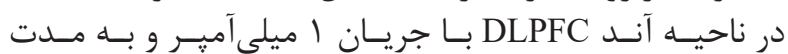

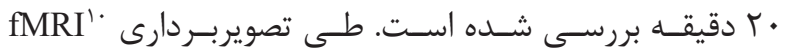

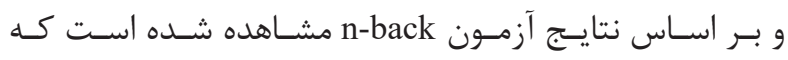

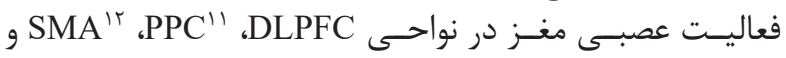

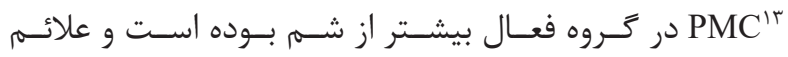

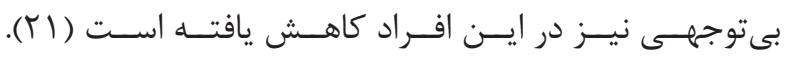

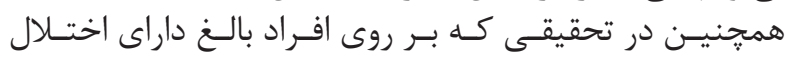

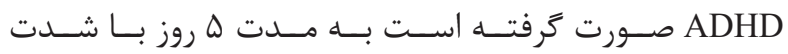

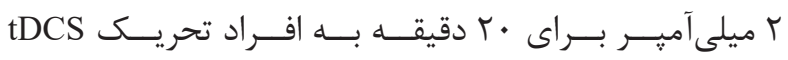

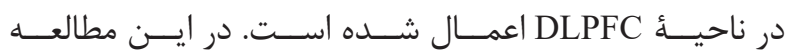

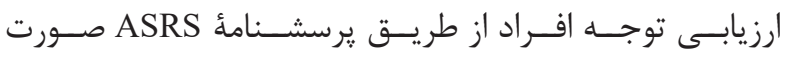

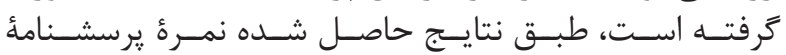

5. Yadollahpour A, Asl HM, Rashidir SJR, Technology. Transcranial direct current stimulation as a non-medication modality for attention enhancement: A review of the literature. Research journal of pharmacy and technology. 2017; 10(1): 311-6.

6. Gladwin TE, den Uyl TE, Fregni FF, Wiers RWJNl. Enhancement of selective attention by tDCS: interaction with interference in a Sternberg task. Neuroscience Letters. 2012; 512(1): 33-7.

7. Coffman BA, Clark VP, Parasuraman RJN. Battery powered thought: enhancement of attention, learning, and memory in healthy adults using transcranial direct current stimulation. NeuroImage. 2014; 85: 895-908.

8. Cachoeira CT, Leffa DT, Mittelstadt SD, Mendes

\footnotetext{
${ }^{8}$ Multiple Object Tracking

${ }^{9}$ Analysis of variance

${ }^{10}$ Functional Magnetic Resonance Imaging

${ }^{11}$ posterior parietal cortex
}

\footnotetext{
${ }^{12}$ supplementary motor area

${ }^{13}$ Pontine micturition center

${ }^{14}$ Executive attention
} 
LST, Brunoni AR, Pinto JV, et al. Positive effects of transcranial direct current stimulation in adult patients with attention-deficit/hyperactivity disorder A pilot randomized controlled study. Psychiatry Research. 2017; 247: 28-32.

9. Cosmo C, Ferreira C, Miranda JGV, do Rosário RS, Baptista A, Montoya P, et al. Spreading effect of tDCS in individuals with attention-deficit/hyperactivity disorder as shown by functional cortical networks: a randomized, double-blind, sham-controlled trial. Child and adolescent psychiatry 2015;6:111.

10. Miler JA, Meron D, Baldwin DS, Garner MJNTatNI. The effect of prefrontal transcranial direct current stimulation on attention network function in healthy volunteers. Neuromodulation: Technology at the Neural Interface. 2018; 21(4): 355-61.

11. Nejati V, Salehinejad MA, Nitsche MA, Najian A, Javadi A-HJJoad. Transcranial direct current $\mathrm{s}$ timulation improves executive dysfunctions in ADHD: implications for inhibitory control, interference control, working memory, and cognitive flexibility. Attention Disorders. 2017; 24(13): 1928-43.

12. Soff C, Sotnikova A, Christiansen H, Becker K, Siniatchkin MJJoNT. Transcranial direct current stimulation improves clinical symptoms in adolescents with attention deficit hyperactivity disorder. Psychiatry and preclinical psychiatric studies. 2017; 124(1): 133-44.

13. Bandeira ID, Guimarães RSQ, Jagersbacher JG, Barretto TL, de Jesus-Silva JR, Santos SN, et al. Transcranial direct current stimulation in children and adolescents with attention-deficit/hyperactivity disorder (ADHD) a pilot study. Child Neurology. 2016; 31(7): 918-24.

14. Roe JM, Nesheim M, Mathiesen NC, Moberget T, Alnæs D, Sneve MHJN. The effects of tDCS upon sustained visual attention are dependent on cognitive load. Neuropsychologia. 2016; 80: 1-8.

15. Kessler RC, Adler LA, Barkley R, Biederman J, Conners CK, Faraone SV, et al. Patterns and predictors of attention-deficit/hyperactivity disorder persistence into adulthood: results from the national comorbidity survey replication. Biological Psychiatry. 2005; 57(11): 1442-51.

16. Mokhtari H, Rabiei M, Salimi SH. Psychometric Properties of the Persian Version ofAdultAttentionDeficit/ Hyperactivity Disorder Self-Report Scale Iranian Journal of Psychiatry and Clinical Psychology. 2015; 21(23): $244-53$.

17. Kessler RC, Adler L, Ames M, Demler O, Faraone S, Hiripi E, et al. The World Health Organization Adult ADHD Self-Report Scale (ASRS): a short screening scale for use in the general population. Psychological Medicine. 2005; 35(2): 245-56.

18. Soltaninejad Z, Nejati V, Ekhtiari HJJoAD. Effect of anodal and cathodal transcranial direct current $s$ timulation on DLPFC on modulation of inhibitory control in ADHD. Attention Disorders. 2019; 23(4): 325-32.

19. Ferrucci R, Bortolomasi M, Vergari M, Tadini L, Salvoro B, Giacopuzzi M, et al. Transcranial direct current stimulation in severe, drug-resistant major depression. Affective Disorders. 2009; 118(1-3): 215-9.

20.Song M, Yun KJN. Beta-frequency EEG activity increased during transcranial direct current stimulation. Cognitive neuroscience and neuropsychology. 2014; 25: 1433-6.

21. Sotnikova A, Soff C, Tagliazucchi E, Becker K, Siniatchkin MJBt. Transcranial direct current stimulation modulates neuronal networks in attention deficit hyperactivity disorder. Brain Topography volume. 2017; 30(5): $656-72$

22. Hanenberg C, Getzmann S, Lewald JJN. Transcranial direct current stimulation of posterior temporal cortex modulates electrophysiological correlates of auditory selective spatial attention in posterior parietal cortex. Neuropsychologia. 2019; 131: 160-70.

23.Lewald JJEbr. Bihemispheric anodal transcranial direct-current stimulation over temporal cortex enhances auditory selective spatial attention. Experimental Brain Research. 2019; 237(6): 1539-49. 